\title{
ATLAS jet trigger performance in 2016 data
}

\author{
Theodor Christian Herwig*, on behalf of the ATLAS Collaboration \\ University of Pennsylvania \\ E-mail: herwig@cern.ch
}

\begin{abstract}
The ATLAS experiment at the LHC uses a two-level trigger system to select events with a predefined topology of interest for future analysis. The hadronic jet trigger is used to select several different topologies containing different types and multiplicities of hadronic jets, thus supporting many different physics searches and measurements. The hadronic jet trigger efficiency for protonproton collision data at a center-of-mass energy of $13 \mathrm{TeV}$ is presented. The efficient selection of events containing hadronic jets requires the characteristics of trigger-level jets and offline jets to be very similar. A comparison of relevant characteristics demonstrates that they are in excellent agreement.
\end{abstract}

38th International Conference on High Energy Physics 3-10 August 2016

Chicago, USA

${ }^{*}$ Speaker. 


\section{Introduction}

During 2016 the LHC provided proton-proton collisions at a center-of-mass energy of $\sqrt{s}=13$ $\mathrm{TeV}$ with instantaneous luminosities reaching beyond $1 \times 10^{34} \mathrm{~cm}^{-2} \mathrm{~s}^{-1}$. This leads to an average of more than 20 interactions occurring in the center of the ATLAS detector per beam crossing, every 25 nanoseconds [1]. The ATLAS trigger system is designed to examine the activity from each crossing and determine which events to record, effectively reducing the rate from the up to $40 \mathrm{MHz}$ frequency to the $1 \mathrm{kHz}$ that is feasible to store for offline reconstruction. The ATLAS trigger is a multi-level system, composed of a Level-1 hardware trigger (L1) and High-Level software Trigger (HLT), which function to reduce the rate in two steps [2].

One goal of the LHC's physics program is to measure Standard Model processes and explore potential New Physics by using hadronic signatures as a probe. The properties of quantum chromodynamics (QCD) specify that the only evidence of colored particle interactions are jets: collimated showers of particles occuring when partons from the hard scatter radiate and hadronize, forming collimated sprays of color-neutral particles. Jets are reconstructed by ATLAS from the energy deposited by these stable hadrons as they pass through the successive layers of the detector. In order to maintain sensitivity to these signatures, the ATLAS jet trigger must be able to reconstruct jets quickly and calculate their energies with a high degree of accuracy [3].

\section{The Level-1 Trigger}

The L1 trigger must be able to reduce the rate of recorded events from the $40 \mathrm{MHz}$ bunch crossing frequency of the LHC to the $100 \mathrm{kHz}$ accepted by the HLT. From 10 to $20 \mathrm{kHz}$ is dedicated to jet signatures. A sliding window algorithm is used to define L1 jets from a grid of jet elements, a block of calorimeter cells spanning $0.2 \times 0.2$ in projective $\eta \times \phi$ space. L1 jets are $4 \times 4$ collections of these jet elements, where the energy in the $2 \times 2$ core is a local maximum [4].

The energy of each trigger tower is calculated after filtering and digitizing the analog pulse and applying pedestal subtraction and noise thresholding. The energies of the constituent towers are then summed to give the energy of each L1 jet. The simplest selection criterion is the single jet selection, where an event containing a jet with $E_{T}$ above threshold is selected and passed to the HLT for further consideration. Multi-jet hypotheses are also employed, with a lower energy threshold required. The efficiency measured in data for the L1 trigger system to select events with jets of a given offline $p_{T}$ is shown in Figure 1 for a variety of items.

\section{The High Level Trigger}

With a lower input rate than L1, the HLT is able to read out cells from the entire calorimeter and topologically cluster them into constituents that can be used as inputs for jet finding in the same manner as in the offline reconstruction [5]. Jet energies obtained by adding constituent momenta are further calibrated in a manner similar to the procedures employed for offline-reconstructed jets [6]. Area-based pileup suppression is applied and jet energies are corrected to constituent scale with the aid of Monte Carlo simulation. Despite not having access to the full set of information used in the offline calibration (such as vertices and tracks), the energies of jets found in the HLT agree very well with corresponding offline jets, resulting in a narrow turn-on region of the efficiency curve. 

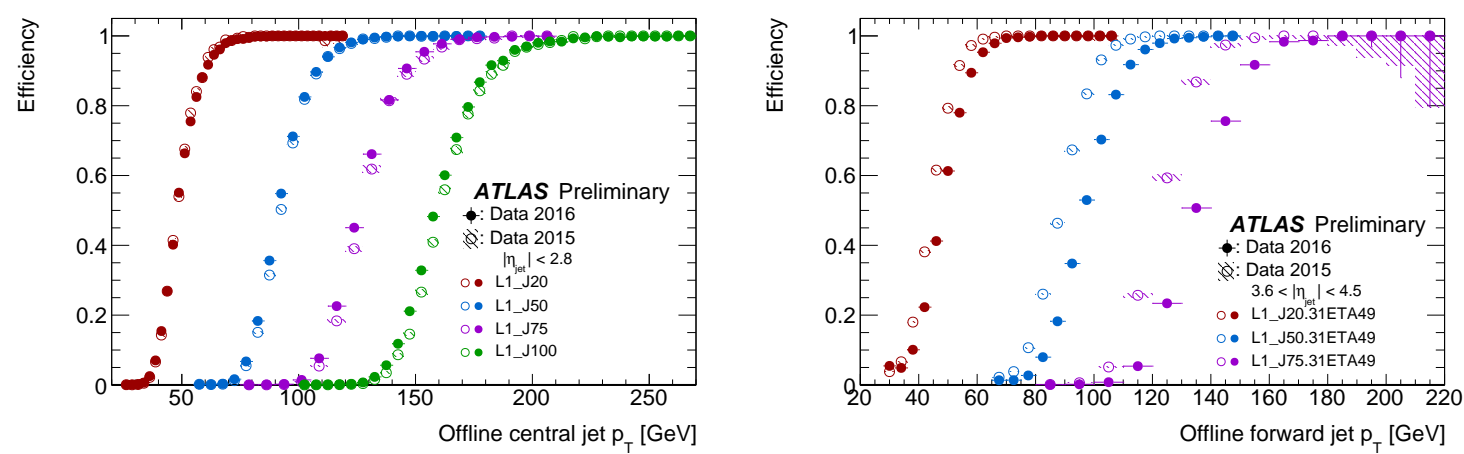

Figure 1: Efficiencies for L1 central $(|\eta|<2.8$, at left) and forward (3.6 $<|\eta|<4.5$, at right) single-jet triggers, shown as a function of leading offline jet $p_{T}$ [7].

The HLT has selections that require at least one jet above threshold for small-radius, largeradius, and reclustered jets. Also recorded are events which contain multiple small-radius jets that pass a looser energy requirement, as well as events with a large $\sum E_{T}$ over all jets in the event. Triggers with lower L1 and HLT thresholds are also operated for use in performance studies, though only a fraction of the large number of passing events are recorded. Figure 2 shows the efficiency of the HLT to select events containing anti- $k_{t} R=0.4$ jets as a function of offline jet $p_{T}$ for an array of single-jet triggers.
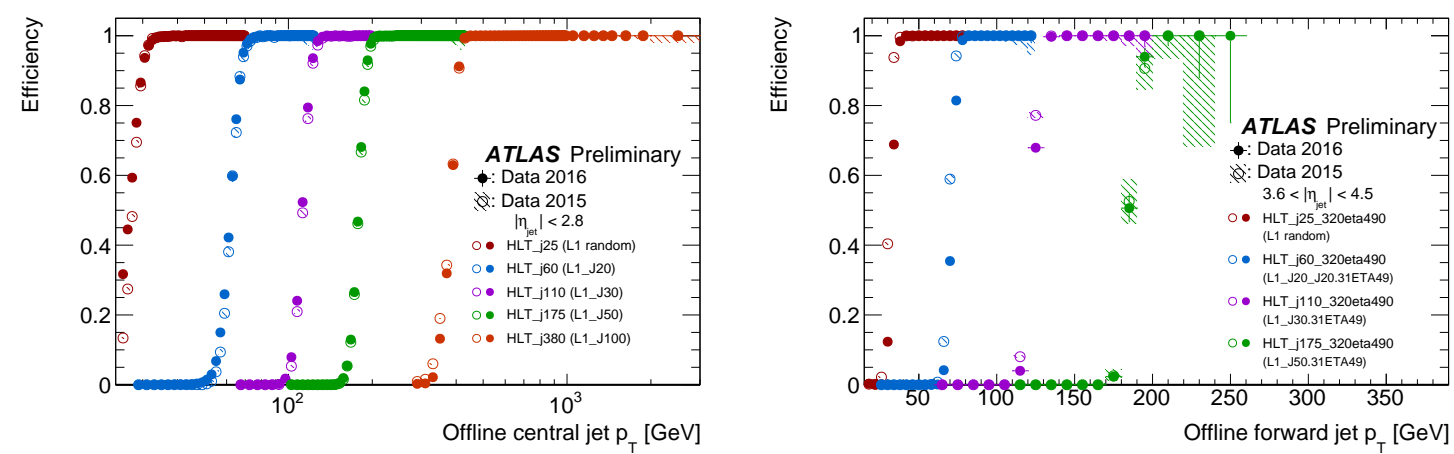

Figure 2: Efficiencies for HLT central (left) and forward (right) single-jet triggers, shown as a function of leading offline jet $p_{T}[7]$.

\section{Recent Developments in Trigger Jet Calibration}

Because of the fundamentally limited computing resources of the trigger there are some algorithms used for offline jet energy calibration that are not currently employed in the HLT. Consequently, HLT jet energies do not exactly match offline, introducing a resolution effect that broadens the turn-on region of efficiency curves. In order to reduce these differences, two additional calibrations were studied in 2016 that will allow for improved trigger efficiency in 2017.

While Monte Carlo can give an excellent first approximation of the jet response, a data-driven calibration is useful to correct jet energies in the forward, more difficult to model, region of the 
detector [8]. In 2016, this $\eta$-intercalibration was derived specifically for HLT jets by balancing a forward jet with a central reference object.

Jet response has also been found to vary significantly depending on the flavor of the parton initiating the jet and the hadron content of its particle shower. The Global Sequential (GS) calibration corrects for these differences using calorimeter shape information and jet track characteristics [9]. Trigger items with both $\eta$ and GS corrections were commissioned in summer 2016 and will become standard in 2017 data-taking. In particular, the complete installation of the Fast TracKer (FTK) will allow for widespread use of track-based corrections in the jet trigger [10].

\section{Summary}

We have presented an overview of the ATLAS jet trigger system, detailing the jet reconstruction and calibration procedure in L1 and HLT systems. The jet trigger performance in 2016 is found to demonstrate excellent agreement between trigger-level and offline jets.

\section{References}

[1] ATLAS Collaboration, The ATLAS Experiment at the CERN Large Hadron Collider, JINST 3 (2008) S08003.

[2] ATLAS Collaboration, ATLAS high-level trigger, data-acquisition and controls: Technical Design Report, ATLAS-TDR-16, CERN-LHCC-2003-022 (2003), URL: http://cds.cern.ch/record/ 616089.

[3] ATLAS Collaboration, The performance of the jet trigger for the ATLAS detector during 2011 data taking, Eur. Phys. J. C 76 (2016) 526, arXiv:1606.07759 [hep-ex].

[4] R. Achenbach et al., The ATLAS Level-1 Calorimeter Trigger, JINST 3 (2008), ATL-DAQ-PUB2008-001, URL: http://cds.cern.ch/record/1080560.

[5] ATLAS Collaboration, Topological cell clustering in the ATLAS calorimeters and its performance in LHC Run 1, arXiv:1603.02934 [hep-ex].

[6] ATLAS Collaboration, Jet energy measurement and its systematic uncertainty in proton-proton collisions at $\sqrt{s}=7 \mathrm{TeV}$ with the ATLAS detector, Eur. Phys. J. C 75 (2015) 75:17, arXiv:1406.0076 [hep-ex].

[7] https://twiki.cern.ch/twiki/bin/view/AtlasPublic/JetTriggerPublicResults.

[8] ATLAS Collaboration, Monte Carlo Calibration and Combination of In-situ Measurements of Jet Energy Scale, Jet Energy Resolution and Jet Mass in ATLAS, ATLAS-CONF-2015-037, URL:https://cds.cern.ch/record/2044941.

[9] ATLAS Collaboration, Jet global sequential corrections with the ATLAS detector in proton-proton collisions at $\sqrt{s}=8 \mathrm{TeV}$, ATLAS-CONF-2015-037, URL:https://cds.cern.ch/record/2001682.

[10] ATLAS Collaboration, Fast TracKer (FTK) Technical Design Report, ATLAS-TDR-021, CERN-LHCC-2013-007 (2013), URL: https://cds.cern.ch/record/1552953. 